\title{
Die Rolle der Sozialwissenschaften in den Gesundheitsberufen
}

\author{
Dominique Sprumont ${ }^{a}$, Theresa Scherer ${ }^{b}$, Settimio Monteverde ${ }^{c}$, Marie-Noelle Kerspern ${ }^{d}$ \\ ${ }^{a}$ Prof. Dr. iur., Université de Neuchâtel, Institut de Droit de la Santé; ${ }^{b}$ MME, RN, Berner Fachhochschule, Fachbereich Gesundheit; \\ c PhD, MME, MAE, RN, Berner Fachhochschule, Fachbereich Gesundheit und Universität Zürich, Institut für Biomedizinische Ethik und Medizingeschichte; \\ d Haute-Ecole Specialisée de Fribourg, Division de Santé
}

\section{Tun und verstehen, was man tut}

Schon ein oberflächlicher Blick in die Curricula der Medizin, der Pflege, der Hebammenkunde oder der Physiotherapie etc. belegt, dass nebst den Fächern, die für das professionelle Handeln «instrumentell» - und deswegen auch unverzichtbar - sind, auch andere Disziplinen den klassischen Fächerkanon heute prägen: die Geistes-, Human- und Sozialwissenschaften im Gesundheitswesen (GHS). Sie leisten einen wichtigen Beitrag für das Verständnis dieses Handelns, decken Zusammenhänge in der Entwicklung professioneller Identität [1] sowie grundlegende ethische und rechtliche Orientierungen therapeutischen Handelns auf. Sie bieten Einblicke in das Erleben und Verarbeiten von Krankheit aus der Perspektive der Psychologie, der Philosophie, der Literatur oder der bildenden Künste [2]. Seit gut vier Jahrzehnten bezeichnet der Mantelbegriff der «Medical Humanities» die Bemühung, den Beitrag dieser Disziplinen besser zu verstehen [3, 4]. Hinreichend belegt ist aber, dass bei der Integration dieses Wissens in Studium und Praxis auch konzeptuelle und praktische Herausforderungen bestehen [5-8].

\section{Eine Schweizer Studie zu den «Medical Humanities»}

In der Schweiz haben die Schweizerischen Akademien der Geistes- und Sozialwissenschaften (SAGW) und der Medizinischen Wissenschaften (SAMW) wesentlich zur Sensibilisierung für die Thematik beigetragen. Von grosser Bedeutung ist der 2012 von den Akademien herausgegebene Bericht "Medical Humanities in der Schweiz» [9]. Dieser beschreibt die Relevanz des Themas und zeigt Prioritäten für die zukünftige Entwicklung. Er wirft auch Fragen auf, die den Anlass zum vorliegenden Projekt gebildet haben: allen voran, welche Disziplinen zu den «Medical Humanities» zu zählen sind und wie relevant diese für das Studium und die Praxis der Gesundheitsberufe sind. Ein Studienteam aus Mitgliedern der Universität Neuchâtel, der Berner
Fachhochschule und der Hochschule Freiburg (CH) nahm sich in einer explorativen Studie dieser Frage an [10]. Grundlage dafür bildete eine Befragung von Fachpersonen im Gesundheitswesen. Aufgrund einer Literaturrecherche und einer Expertenbefragung wurde ein Fragebogen erstellt, von der deutschen in die französische Sprache übersetzt, rückübersetzt und validiert. Die Daten wurden mittels eines zweisprachigen Fragebogens im Frühjahr 2015 online mittels Surveymonkey ${ }^{\circledR}$ erhoben. Die Teilnehmenden wurden durch Ankündigung in einschlägigen Fachmedien rekrutiert.

\section{Relevant, aber altersabhängig}

486 Personen haben den Fragebogen bis zum Ende beantwortet. Sowohl bezüglich der Sprachregion, der Berufe als auch des Geschlechts kann die Stichprobe als repräsentativ für den Schweizer Kontext betrachtet werden. Während der erste Teil des Fragebogens allgemeine Wahrnehmungen zu GHS in der Medizin erhob, war der zweite Teil nur für diejenigen bestimmt, welchen der Begriff der "Medical Humanities» vertraut war $(\mathrm{n}=196)$.

(1) Rolle und Bedeutung der Geistes-, Human- und Sozialwissenschaften im Gesundheitswesen (GHS): Insgesamt konnten bezüglich der Berufszugehörigkeit vier Untergruppen gebildet werden: Pflegeberufe $(n=306)$, Arztberuf $(n=65)$, weitere Gesundheitsberufe $(n=46)$ und weitere Berufe ( $n=69)$ [10].

Hinsichtlich der Frage, welche Disziplinen der GHS für die Ausbildung und Praxis der Gesundheitsberufe unerlässlich sind, fällt die hohe Zustimmung für die Ethik auf. Mit Hilfe logistischer Regression wurde untersucht, ob ein Zusammenhang zwischen der Wahl einer bestimmten Disziplin und anderen Variablen besteht. Für Ethik, Anthropologie, Soziologie und Geschichte konnte ein Einfluss der Variable Alter der Befragten nachgewiesen werden. Auf die Frage nach dem Ziel der Vermittlung der GHS im Studium von Gesundheitsfachpersonen äusserten die Teilnehmenden die höchste Zustimmung zur Persönlichkeitsentwicklung 
der Studierenden und zu ihrer Orientierung an den Bedürfnissen der Patienten.

(2) GHS im Unterricht: Insgesamt gaben 48 Teilnehmende an, in einer Disziplin der GHS lehrend tätig zu sein. Sie zeichnen ein eher kritisches Bild über die Akzeptanz der GHS innerhalb des Kollegiums und im Ausbildungsprogramm, dies im Gegensatz zu dem hohen Stellenwert, den die Studierenden ihnen - in den Augen der Unterrichtenden - beimessen. Insgesamt belegen die Ergebnisse dieser explorativen Studie, dass den GHS ein hoher Stellenwert beigemessen wird. Hinsichtlich der curricularen Verankerung zeigen sie aber auch Widerstände, die explizit zum Gegenstand eines Faculty Development werden müssen. Angesichts der Beobachtung, dass mit steigendem Alter der Teilnehmenden auch die Zustimmung über die Relevanz der GHS für die Praxis steigt, bedarf es weiterer Forschung, wie die GHS über das Grundstudium hinaus kontinuierlich thematisiert resp. im Rahmen der klinischen und berufsbegleitenden Fort- und Weiterbildung optimal verteilt werden können.

\section{Ein interdisziplinäres Verständnis therapeutischen Handelns}

Fachpersonen im Gesundheitswesen arbeiten heute unter Bedingungen eines erhöhten Leistungs- und Ressourcendrucks, der durch die sozioökonomischen Gradienten im Zugang zu Gesundheitsleistungen verstärkt wird [11]. Eine Stärkung der Reflexionsbereitschaft und der Reflexionsfähigkeit stellt deshalb ein vorrangiges Ziel der Aus-, Fort- und Weiterbildung in den Gesundheitsberufen dar, die Fachpersonen befähigt, diesem Druck standzuhalten. Die Geistes-, Human- und Sozialwissenschaften bieten optimale Grundlagen dazu. Als "frischer Wind» im Fächerkanon der Gesundheitsberufe und in der Fort- und Weiterbildung leisten diese Disziplinen einen Beitrag zu einer wirksamen Patientenversorgung und zur Erreichung der sozialen Ziele der Medizin, aber auch zur psychischen und moralischen Resilienz von Fachpersonen. Für einen solchen interdisziplinären Zugang auf das Handeln der Gesundheitsberufe bedarf es eines breit angelegten Verständnisses der GHS, das die interprofessionelle Zusammenarbeit fördert. Dieses schenkt auch denjenigen Populationen Aufmerksamkeit, die unter sich verändernden gesundheitspolitischen Rahmenbedingungen vulnerabel sind. Ob das Konzept der «Medical Humanities» dies zu leisten vermag, kann aufgrund der Ergebnisse dieser explorativen Studie nicht beantwortet werden. Sie liefert aber Hinweise dafür, dass ein Handlungsbedarf besteht hinsichtlich einer prominenteren Platzierung der GHS in den Curricula der Gesundheitsberufe. Dazu bedarf es weiterer Forschung. Diese soll Wege aufzeigen, wie zukünftige Fachpersonen im Gesundheitswesen durch eine optimale Integration der GHS auf die zunehmend komplexen Aufgaben der beruflichen Praxis vorbereitet werden können.

\section{Finanzierung}

Das vorliegende Projekt wurde durch die Schweizerische Akademie der Geistes- und Sozialwissenschaften (SAGW) unterstützt.

Literatur

1 Schwartz A, Abramson J, Wojnowich I, Accordino R, Ronan E, Rifkin $M$, Mary R. Evaluating the Impact of the Humanities in Medical Education. Mt Sinai J Med. 2009;76(4):372-80. DOI: 10.1002/ msj.20126.

2 Cole T, Carlin N, Carson R. Medical Humanities. An Introduction. Cambridge: Cambridge University Press; 2015.

3 Walsh $\mathrm{K}$. The humanities in medical education - what is the future? Ann Ist Super Sanita. 2013;49(2):107.

4 Meakin R. Teaching medical students professionalism: what role for the medical humanities? Med Humanit. 2007;33(2):105

5 Doukas J, McCullough L, Wear S. Medical Education in Medical Ethics and Humanities as the Foundation for Developing Medical Professionalism. In: Academic Medicine. 2012;87(3):334-41. DOI: 0.1097/ACM.Ob013e318244728c

6 Pattison S. Medical Humanities: a vision and some cautionary notes. Med Humanit. 2003;29(1):33-6. DOI: 10.1136/mh.29.1.33.

7 Downie R. Commentary to: Medical humanities: a vision and some cautionary notes. Med Humanit. 2003;29(1):37-8. DOI: 10.1136/ mh.29.1.37.

8 Ousager J, Johannessen H. Humanities in Undergraduate Medical Education: A Literature Review. Acad Med. 2010;85(6):988-98. DOI: 10.1097/ACM Ob013e3181dd226b.

9 Akademien der Wissenschaften Schweiz (2012). Medical Humanities in der Schweiz. Bern: Akademien der Wissenschaften Schweiz. Zugänglich unter: http://www.akademien-schweiz.ch/ $\mathrm{dms} / \mathrm{D} /$ Publikationen/Berichte/MedicalHumanities_d.pdf).

10 Sprumont D, Scherer T, Monteverde S, Kerspern MN (2017). Les sciences sociales dans les professions de la santé, in: Jusletter 23. Oktober 2017. Zugänglich unter: https://jusletter.weblaw.ch/ juslissues/2017/911/les-sciences-sociale_148a9c7dOc.html (Abruf am 24.10.2017)

11 Sharma M, Pinto A, Kumagai A. Teaching the Social Determinants of Health: A Path to Equity or a Road to Nowhere? Acad Med. 2017; Apr 25. DOI: 10.1097/ACM.0000000000001689 [Epub ahead of print]. 